\title{
Percepção do estudante de enfermagem em relação a visita domiciliária
}

\author{
Perception of the nursing student about house visiting
}

Percepción del estudiante de enfermería en relación a la visita domiciliaria

Renato Pereira Neto ${ }^{1}$; Michele Luciana Sena Tomé1; Vanessa Aparecida Ballista Tavares de Araújo'; Diego Santiago Montandon" ${ }^{1 *}$.

\section{RESUMO}

Objetivo: Compreender a percepção do estudante de enfermagem acerca da visita domiciliária. Método: Estudo método descritivo de abordagem qualitativa, que utilizou a técnica de coleta de dados com entrevistas semiestruturadas com 28 estudantes de uma universidade do interior do estado de São Paulo, os dados foram submetidos à análise de conteúdo de Bardin. Resultados: Diante deste estudo fica evidente que os estudantes reconhecem a visita como um potente instrumento de cuidado integral à saúde. Contudo grande parte das ações desenvolvidas pelos estudantes possui uma abordagem estritamente tecnicista e biologicista, seguindo o modelo de atenção à saúde biomédico hegemônico. Conclusão: Os estudantes possam refletir sobre sua prática e passem a planejar as visitas ou o ensino da mesma voltados ao cuidado integral do indivíduo mesmo quando realizadas apenas a usuário portadores de patologias, a fim de evitar o cuidado reducionista voltado apenas ao problema.

Palavras-chave: Visita Domiciliar, Enfermagem, Atenção Primária de Saúde, Estudantes.

\begin{abstract}
Objective: To understand the perception of the nursing student about the home visit. Method: A descriptive method of qualitative approach, which used the technique of data collection with semi-structured interviews with 28 students from a university in the interior of the state of São Paulo, the data were submitted to Bardin content analysis. Results: In the face of this study, it is evident that the students recognize the visit as a powerful instrument of integral health care. However, most of the actions developed by the students have a strictly technical and biological approach, following the hegemonic biomedical health care model. Conclusion: Students can reflect on their practice and start planning their visits or teaching them to the integral care of the individual even when performed only to users suffering from pathologies, in order to avoid reductionist care focused only on the problem.
\end{abstract}

Keywords: Home visit, Nursing, Primary Health Care, Students.

\section{RESUMEN}

Objetivo: Comprender la percepción del estudiante de enfermería acerca de la visita domiciliaria. Método: Estudio método descriptivo de abordaje cualitativo, que utilizó la técnica de recolección de datos con entrevistas semiestructuradas con 28 estudiantes de una universidad del interior del estado de São Paulo, los datos fueron sometidos al análisis de contenido de Bardin. Resultados: Ante este estudio queda claro

${ }^{1}$ Faculdade de Enfermagem de Presidente Prudente da Universidade do Oeste Paulista, Presidente Prudente - SP. *E-mail: diegosantiago@unoeste.br

SUBMETIDO EM: 11/2018

ACEITO EM: 12/2018

PUBLICADO EM: 02/2019 
que los estudiantes reconocen la visita como un potente instrumento de cuidado integral a la salud. Sin embargo, gran parte de las acciones desarrolladas por los estudiantes tienen un enfoque estrictamente tecnicista y biologicista, siguiendo el modelo de atención a la salud biomédica hegemónica. Conclusión: Los estudiantes puedan reflexionar sobre su práctica y pasar a planificar las visitas o la enseñanza de la misma dirigidas al cuidado integral del individuo incluso cuando se realizan sólo a usuarios portadores de patologías, a fin de evitar el cuidado reduccionista orientado sólo al problema.

Palabras clave: Visita Domiciliaria, Enfermería, Atención Primaria de Salud, Estudiantes.

\section{INTRODUÇÃO}

A atuação do Enfermeiro na Estratégia Saúde da Família (ESF) requer amplo envolvimento comunitário para efetivar ações que melhorem as condições de saúde populacional. Neste sentido a ferramenta de maior impacto para este profissional é a visita domiciliar (VD), no qual são estabelecidos vínculos profissionais de grande importância para o direcionamento do cuidado integral (AZEVEDO et. al., 2017).

O trabalho dos Enfermeiros na ESF é voltado para a assistência integral e contínua de todos os membros da família da área de abrangência, em cada fase de seu ciclo de vida, sem perder de vista seu contexto familiar e social. Para atuar sobre um sistema familiar é necessário estabelecer vínculo e acesso à família. Prestar assistência a uma família desconhecendo seus antecedentes e estilo de vida é o mesmo que relacionar-se sem a devida apresentação (GUIMARÃES et. al., 2017).

Historicamente, o Programa Saúde da Família, implantado pelo Ministério da Saúde em 1994, sob a égide da discussão da reformulação do sistema de saúde, trouxe uma nova dinâmica, estabelecendo uma relação de vínculo com a comunidade, humanizando a prática na perspectiva dos profissionais envolvidos, fortalecendo os princípios do Sistema Único de Saúde (SUS) de universalidade, acessibilidade, equidade e integralidade que dispõe de ações de promoção de saúde, prevenção de riscos/agravos e assistência ao doente (OLIVEIRA FIGUEIREDO et. al., 2017).

Hoje a ESF consiste em uma estratégia que possibilita a integração e promove a organização das atividades em território definido, com o objetivo de propiciar o enfrentamento e resolução dos problemas, promovendo ações aos sujeitos e suas famílias de forma individual, integral e contínua (LACERDA et. al., 2017).

Nesse sentido, é necessário utilizar ferramentas que permitam conhecer toda a família para assim, se propor intervenção adequada. Visando à produção de novos modos de cuidado a ESF propõe a VD como instrumento central no processo de trabalho das equipes, a pratica da VD determina o rompimento do padrão de assistência focado na doença, constituindo em um importante instrumento de atenção à saúde que oportuniza a partir do conhecimento da realidade local, do indivíduo e de sua família, o fortalecimento dos vínculos entre paciente e profissional e a adesão ao tratamento, além de atuar na promoção de saúde, prevenção, tratamento e reabilitação de doenças e agravos (ARANTES et. al., 2016).

Uma das principais limitações do enfermeiro no que se refere a atuação na VD é a escassez de programas de capacitação e isso pode ser reflexo da formação do mesmo. A atuação em VD com pouco preparo para essa modalidade de atenção, implica em apresentação de dificuldades em identificar as demandas necessárias e propor intervenções junto aos indivíduos e suas famílias. Além disso, a possibilidade de causar dependência a população, pois pode promover acomodação e desestímulo a procurar a unidade de saúde (SOSSAI, PINTO; 2010).

Para que o enfermeiro realize a VD de maneira satisfatória e para que esteja apto a extrair as potencialidades e dificuldades da realização da VD, é necessário que sua formação contemple esta temática em seu currículo. Assim torna-se importante compreender a percepção do estudante de enfermagem acerca da VD. Sendo assim, o objetivo do presente estudo é compreender a percepção do estudante de Enfermagem acerca da Visita Domiciliária. 


\section{MÉTODOS}

O presente trabalho trata-se de um estudo descritivo-exploratório com abordagem qualitativa (MINAYO, GUERRIERO; 2014). Os dados foram tratados conforme análise temática de conteúdo, que permite ao pesquisador liberdade e inovação no momento de analisar as entrevistas. Essa técnica segue três etapas: pré- análise, exploração do material e tratamento dos resultados com a inferência e interpretação (BARDIN, 1991).

Tendo em vista o currículo acadêmico do curso de graduação no qual estudam os sujeitos de pesquisa, optou-se pelo modelo de seleção proposital, incluindo estudantes concluintes por já terem imersão prática no cenário da ESF. Neste contexto, todos os estudantes concluintes aceitaram participar desta pesquisa e assinaram o Termo de Consentimento Livre e Esclarecido. Para a execução da coleta de dados, foi realizado um contato prévio com os pesquisados com a finalidade de explicar os objetivos da pesquisa.

Com isso foi agendado o melhor horário para as entrevistas individuais, que duraram em média quinze minutos e ocorreram em uma sala de aula no campus da universidade, apenas com um dos pesquisadores de forma reservada para garantir a ausência de constrangimento e desconfortos, sendo solicitado autorização para gravar as entrevistas que posteriormente foram transcritas.

Foram entrevistados 28 estudantes concluintes do curso de graduação em enfermagem, sendo 24 do sexo feminino e 04 do sexo masculino. As idades variaram entre 21 e 41 anos, sendo a idade média de 27 anos. A coleta de dados foi realizada em março de 2017, e o roteiro para entrevista foi constituído de duas partes: a primeira com os dados de identificação do entrevistado com variáveis relacionadas com a idade e sexo; na segunda parte com três perguntas norteadoras e 13 questões de apoio que foram utilizadas em caso de discursos sucintos com o interesse de responder ao objetivo desta pesquisa.

A partir das leituras e análises dos discursos que foram transcritos, realizou-se a compilação dos dados, identificação dos núcleos de sentido comuns e foram elencadas duas categorias com depoimentos que as representam e as inferências construídas por meio da análise temática do conteúdo. A saber: Categoria 1 A realização da visita domiciliária seguindo um modelo biomédico hegemônico; Categoria 2 - A Visita Domiciliária como instrumento de formação de vínculo.

Para garantir o sigilo e anonimato durante a tabulação e exposição das falas dos entrevistados, optou-se por utilizar a letra "E" e o número de sequência que ocorreram as entrevistas ao utilizar suas falas durante os resultados e discussões. Para realização da coleta de dados, foi realizado um contato prévio com os pesquisados com a finalidade de explicar os objetivos da pesquisa e os estudantes que aceitaram participar da mesma, assinaram o termo de consentimento livre esclarecido.

Este estudo cumpriu as normas éticas vigentes na Resolução 466/2012 do Conselho Nacional de Saúde, sendo autorizado pelo Comitê de Ética em pesquisa sob o número 3481 e ao CAAE 59977416.0.0000.551.

\section{RESULTADOS E DISCUSSÃO}

A seguir apresenta-se as categorias vislumbras e suas inferências diante da literatura sobre a temática.

Categoria 1 - A realização da visita domiciliária seguindo um modelo biomédico hegemônico.

A visita domiciliária é uma prática no curso de graduação em enfermagem, quando o estudante passa pelos campos de prática e estágio supervisionado na Atenção Primária. Pode-se identificar que a mesma fez parte da vida acadêmica dos estudantes e constatou-se que, grande parte das ações desenvolvidas pelos estudantes possuía uma abordagem estritamente tecnicista e biológica, seguindo o modelo de atenção a saúde biomédico hegemônico, fato observado em 21 das 28 entrevistas, podendo ser evidenciado em alguns discursos como: 
"[...] se tinha curativo fazia o curativo, se o paciente era diabético fazia um controle glicêmico, controle de pressão isso ai [...]". E10

"[...] então a gente ia com a finalidade de prestar o cuidado com as pessoas que não tinha como se locomover ao posto [...]". E20

"[...] as vezes fazer curativo porque era muito precário e o cuidados não sabia [...]". E18

Nos discursos é possível identificar que a visita domiciliária é uma extensão das ações da unidade voltadas a atender indivíduos doentes, debilitados e que necessitam de procedimentos, a ênfase no modelo hegemônico faz com que o estudante perca a oportunidade de realizar o cuidado integral e voltado à família.

Fertonani e colaboradores defendem que o modelo biomédico hegemônico tem estreita relação com a natureza biológica da origem das doenças e faz com que o profissional tenha assistência voltada a cura das doenças e, por conseguinte maiores investimentos públicos na atenção secundária e terciária. Neste sentido, verifica-se pouca ênfase no processo saúde-doença e ausência do olhar diante dos aspectos culturais, sociais e ambientais das pessoas dificultando 0 entendimento multifatorial das dimensões humanas.

Embora seja um modelo que permite uma abordagem direta sobre o doente, é uma explicação que, se solitária, torna-se bastante reducionista, não se preocupando nem com o contexto social, nem emocional em que estas condições podem ocorrer (AZEVEDO et. al., 2017/ FERTONANI et. al., 2015).

Contudo vale ressaltar que apesar da grande maioria dos entrevistados apontarem a utilização da VD como extensão das ações da unidade, entre os entrevistados encontra-se de forma recorrente, contradizendo esta afirmação, discursos que destacam a VD como importante ferramenta no cuidado integral aos usuários.

"[...] a gente via tudo isso, as condições psicossocial, ambiente, recursos financeiros e humanos, tudo isso a gente tinha que observar além do que a gente estava indo fazer [...]". E18

"[...] cuidado integral onde você conhece a pessoa como um todo não somente a parte clínica que ela chega no posto [...]" E1I

"[...] a partir da visita domiciliária a partir de um olhar clínico identificar as necessidades do paciente a ser atendido e acompanhado dentro da atenção primária [...]". E1

As VDs se destacam como intervenção capaz de promover ações preventivas, curativas, de promoção e de reabilitação dos indivíduos em seu contexto familiar por permitir uma maior aproximação dos profissionais com a realidade de vida e dinâmica das famílias. O papel da enfermagem, desse modo não implica unicamente em lidar com estados de saúde da família, mas também interagir com situações que respaldem a integridade familiar. Assim, deve reconhecer e entender como a saúde de cada membro da família afeta a unidade familiar e também a importância dessa unidade sobre a saúde de cada indivíduo da família (GOMES, FRACOLLI; 2015).

Para construir uma pratica pautada na superação de um modelo centralizado e curativo, a formação do enfermeiro apresenta uma trajetória de mudança, entretanto nota-se que ainda existe uma grande dicotomia no que se refere a articulação entre a teoria e a pratica (GUIMARÃES et. al., 2017). Grande parte dos estudantes apesar de compreenderem a real finalidade da VD ainda a realizam com uma abordagem estritamente tecnicista e biologicista.

Portanto, apenas a inclusão de novas diretrizes e de modo consequente novas estruturas curriculares, não permite o desenvolvimento de profissionais críticos, reflexivos e transformadores da realidade, há uma necessidade de mudança na visão dos próprios docentes associados ao papel da universidade na comunidade, proporcionando uma proximidade entre o estudante e esta, pelo desenvolvimento nesse aluno de aptidões que transcendem aspectos técnicos e biológicos (CAETANO et. al., 2006). 
Categoria 2 - A visita domiciliária como instrumento de formação de vínculo.

Fica evidente nesta categoria que o estudante reconhece a visita domiciliária como um potente instrumento de cuidado integral e para isso o estabelecimento de vínculo é essencial. Nesse contexto, evidenciamos a mesma percepção do estudante em 16 das 28 entrevistas analisadas, destacando os seguintes discursos:

"Você como profissional visitando uma família, você pode construir um vínculo, e desta forma achar uma maneira adequada de promover a saúde no seu território [...]". E3

"Fortalecimento de vínculo, pois na ESF se fala muito de vínculo e somente na visita domiciliar podemos observar e estabelecer esse vínculo". E21

Apesar dos estudantes destacarem que realizavam a visita com finalidade curativa e assistencial, no que diz respeito à realização de procedimentos, os mesmos expressam, que a visita é essencial para o cuidado e que permite este contato mais próximo com o usuário possibilitando a criação de vínculo entre eles e consequentemente a sensibilização com a situação do outro, viabilizando a construção de um processo de transferência entre o usuário e o trabalhador de saúde que possa servir para o desenvolvimento de projetos terapêuticos individualizados.

A formação de vínculo coma as unidades familiares representa uma das diretrizes da ESF. No campo da saúde pública, o vínculo representa uma união entre os conceitos de humanização, responsabilização, acolhimento e integralidade. Neste contexto, a VD, constitui-se como um momento rico de troca de informações entre usuário e profissional de enfermagem, no qual se estabelece a dinâmica das relações, ou seja, a escuta qualificada, o vínculo e o acolhimento. A prática da VD é um dos instrumentos essenciais para o entendimento das condições que afetam positiva ou negativamente a vida do usuário (DA SILVA et. al., 2017).

Em relação ao acompanhamento feito à comunidade por meio da VD, percebe-se que parte dos alunos entrevistados, entende essa prática como um elemento importante para a criação e desenvolvimento de vínculo com as famílias.

Em análise das mesmas entrevistas podemos encontrar discursos que destacam além do vínculo o cuidado integral.

\section{"[...] Cuidado integral onde você conhece a pessoa como um todo não somente a parte clínica} que ela chega ao posto [...]". E21

O cuidado integral é percebido na fala dos estudantes, ou seja reconhecem que o cuidado não pode mais ser reducionista, meramente curativo, pois os problemas de saúde não se resumem apenas a alterações físicas ou orgânicas. O indivíduo é influenciado pelo meio social em que vive e trás consigo representações que influenciam a forma como adoece e se recupera e cabe ao enfermeiro reconhecer suas necessidades e vulnerabilidades para prestar o cuidado integral e isso só é possível por meio do vínculo (LACERDA et. al., 2017).

Para que haja a integralidade no cuidado à saúde é necessário viabilizar, em cada caso, uma intervenção que atenda da melhor forma às necessidades do indivíduo, e determinar uma prática de saúde considerando o processo saúde-doença e a pessoa introduzida nesse processo, que é influenciado por convicções, crenças, valores, situação econômica e social (SILVA, DE SENA; 2008).

É importante elucidar que este estudo apresenta como limitação o fato de ter como sujeitos, pessoas oriundas de uma mesma instituição e grupo acadêmico, impossibilitando comparações interinstitucionais. Todavia destaca-se a relevância em apresentar a percepção destes futuros profissionais diante de uma atuação focal que interfere diretamente na qualidade da atenção à saúde prestada a população. 


\section{CONSIDERAÇÕES FINAIS}

Diante da inquietação deste estudo e dos resultados encontrados, considera-se que os estudantes têm a percepção adequada da importância da VD, contudo demonstram práticas desvinculadas com a solidez teórica, ou seja, existe uma dicotomia entre o saber e fazer na percepção da VD entre estes sujeitos.

Por fim espera-se que com este estudo, profissionais, docentes e estudantes possam refletir sobre sua prática e passem a planejar as visitas ou o ensino da mesma voltados ao cuidado integral do indivíduo mesmo quando realizadas apenas a usuário portadores de patologias, a fim de evitar o cuidado reducionista voltado apenas ao problema.

\section{REFERÊNCIAS}

1. ARANTES LJ, SHIMIZU HE, MERCHÁN-HAMANN E et al. Contribuições e desafios da Estratégia Saúde da Família na Atenção Primária à Saúde no Brasil: revisão da literatura. Ciência \& Saúde Coletiva. 2016; 2(1):1499-1510.

2. AZEVEDO ACDF, OKADA KK, DE LIMA GZ, et al. Visita domiciliar na atenção à saúde mental: relatando a experiência de um projeto de extensão universitária. Rev Uningá. 2017; 52(1):40-43.

3. BARDIN, Laurence. Análisis de contenido. Ediciones Akal, 1991.

4. CAETANO SAC, MUNIZ MJM, SILVA LDFD, et al. Formação do enfermeiro para o cuidado: reflexões da prática profissional. Reben. 2006; 59(6); 805-807.

5. DA SILVA IS, ARBOIT ÉL, DA SILVEIRA MR, et al. Visita domiciliar: estratégia para a promoção da saúde de pacientes crônicos. RevEnf. 2017 12(12): 88-99.

6. FERTONANI HP, PIRES DEPD, BIFF D, et al. Modelo assistencial em saúde: conceitos e desafios para a atenção básica brasileira. Ciência \& Saúde Coletiva. 2015; 20: 1869-1878.

7. GOMES MFP, FRACOLLI RA, MACHADO BC. Et al. Atenção domiciliar do enfermeiro na estratégia saúde da família. O Mundo da Saúde. 2015; 39(4): 470-475.

8. GUIMARÃES MSA, TAVARES NUL, NAVES JDOS, et al. Estratégia saúde da família e uso racional de medicamentos: o trabalho dos agentes comunitários em Palmas (TO). Trab Edu e Saúde. 2017; 15(1): 183-203.

9. LACERDA MKS, PEREIRA ACA, PEREIRA MM, et al. Ferramentas de abordagem familiar: estudo de uma família cadastrada em uma equipe de estratégia saúde da família em Montes Claros, Minas Gerais, Brasil. Rev de Ini Cientí UniCor. 2017; 7(1): 25-34.

10. MINAYO MCS, GUERRIERO ICZ. Reflexividade como éthos da pesquisa qualitativa. Ciência \& Saúde Coletiva. 2014;19(4):11031112.

11. OLIVEIRA FN, JUNIOR RP, GILSON F. et al. Politicas públicas de saúde: aportes para se pensar a trajetória histórica Brasileira. Ver Desafios. 2017; 4(1): 21-31

12. SILVA KL, DE SENA RR. Integralidade do cuidado na saúde: indicações a partir da formação do enfermeiro. REUSP. 2008; 42(1): 48-56.

13. SOSSAI LC, PINTO IC. A visita domiciliária do enfermeiro: fragilidades x potencialidades. Ciência, Cuidado e Saúde. 2010; 9(3): 569-576. 